\title{
Application of Autofluorescence for Confocal Microscopy to Aid in Archaeoparasitological Analyses
}

\author{
Johnica Jo Morrow ${ }^{1,2}$, Christian Elowsky ${ }^{3}$ \\ ${ }^{1}$ Pre-Health Pathways, Student Success Center, South Dakota School of Mines \& Technology, Rapid City, SD 57701, USA; ${ }^{2}$ Pathoecology and \\ Palynology Laboratory, School of Natural Resources, University of Nebraska-Lincoln, Lincoln, NE 68503, USA; ${ }^{3}$ Department of Agronomy and \\ Horticulture, University of Nebraska-Lincoln, Lincoln, NE 68583, USA
}

\begin{abstract}
Confocal laser scanning microscopy (CLSM) was used to examine archaeoparasitological specimens from coprolites associated with La Cueva de los Muertos Chiquitos (CMC) located near present-day Durango, Mexico. The eggs for 4 different types of parasites recovered from CMC coprolites were imaged using CLSM to assist with identification efforts. While some of the parasite eggs recovered from $\mathrm{CMC}$ coprolites were readily identified using standard light microscopy (LM), CLSM provided useful data for more challenging identifications by highlighting subtle morphological features and enhancing visualization of parasite egg anatomy. While other advanced microscopy techniques, such as scanning electron microscopy (SEM), may also detect cryptic identifying characters, CLSM is less destructive to the specimens. Utilizing CLSM allows for subsequent examinations, such as molecular analyses, that cannot be performed following SEM sample preparation and imaging. Furthermore, CLSM detects intrinsic autofluorescence molecules, making improved identification independent of resource and time-intensive protocols. These aspects of CLSM make it an excellent method for assisting in taxonomic identification and for acquiring more detailed images of archaeoparasitological specimens.
\end{abstract}

Key words: Archaeoparasitology, autofluorescence, confocal laser scanning microscopy, parasite eggs, La Cueva de los Muertos Chiquitos

\section{INTRODUCTION}

Confocal laser scanning microscopy (CLSM) is a powerful tool that can be used for studying a variety of organisms [1-10]. Using CLSM is an excellent way to examine the internal and external features of microscopic organisms in detail without compromising the structural integrity of specimens or rendering specimens unusable for further analyses. Such examinations can be useful for taxonomic identifications of archaeoparasitological specimens. Using CLSM techniques for the visualization of parasite eggs does not require staining preparation as these specimens are able to be imaged using the intrinsic properties of the organisms [11]. This is true for both modern and ancient parasite eggs. Herein, we report a novel application of CLSM for the analysis of parasite eggs collected from archaeological source materials. These applications can be applied to future studies of archaeoparasitological materials, in-

- Received 31 July 2019, revised 4 September 2019, accepted 12 October 2019.

*Corresponding author (johnicamorrow@yahoo.com)

(c) 2019, Korean Society for Parasitology and Tropical Medicine

This is an Open Access article distributed under the terms of the Creative Commons Attribution Non-Commercial License (http://creativecommons.org/licenses/by-nc/4.0) which permits unrestricted non-commercial use, distribution, and reproduction in any

medium, provided the original work is properly cited. cluding both helminth eggs and arthropod specimens.

\section{MATERIALS AND METHODS}

The specimens used for this study were obtained from La Cueva de los Muertos Chiquitos (CMC), a cave in Durango, Mexico, which was utilized by the Loma San Gabriel 1,300 years ago. Coprolites (i.e., desiccated feces) were processed from this site for performing standard archaeoparasitological analysis, which traditionally has included stereomicroscopy and light microscopy (LM). After conducting traditional analyses of these samples, some of the parasite eggs were easily recognizable, while others proved to be more challenging to identify. Thus, select slide-mounted parasite egg specimens from CMC coprolites were further subjected to CLSM to aid in identification efforts and to gain high-quality images of representative specimens [12]. To the authors' knowledge, this is the first time that CLSM has been used to image archaeoparasitological specimens and the first time that CLSM has been used to assist in taxonomic identification efforts for parasite eggs recovered from coprolites.

Slides were prepared by adding a small amount of processed material to a drop of glycerin onto a glass microscope slide 
and topping it with a $22 \times 22 \mathrm{~mm}$ glass coverslip. Slides were sealed using a clear, commercial nail lacquer and were selected for CLSM based upon parasite egg preservation and parasite egg concentrations [12]. The selected slides contained multiple

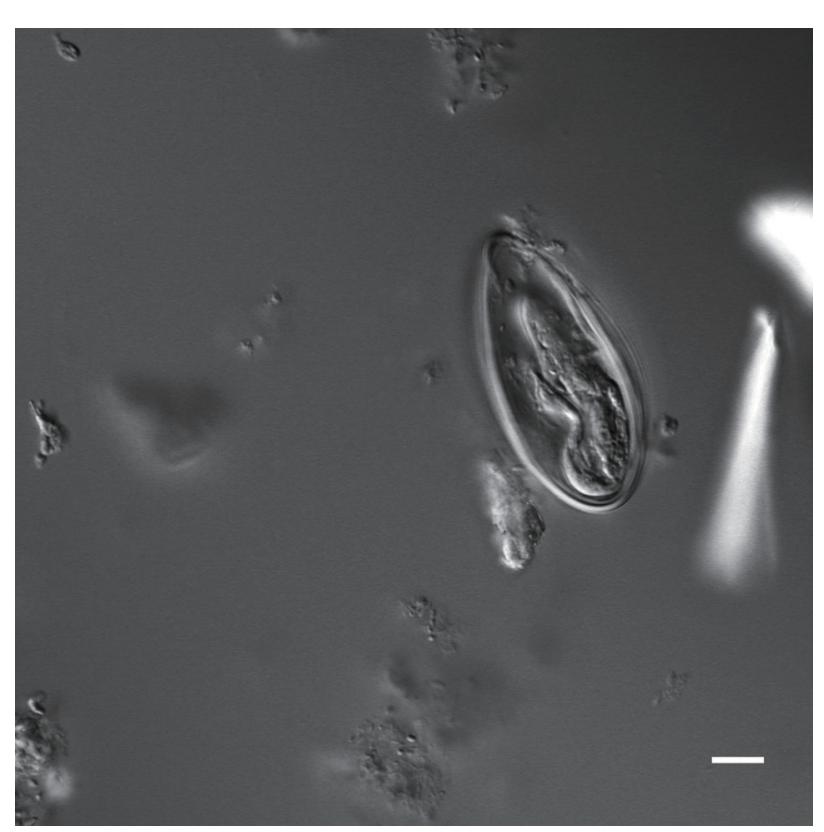

Fig. 1. Nomarski image of Enterobius vermicularis egg recovered from a CMC coprolite (Scale $=25 \mu \mathrm{m})$.

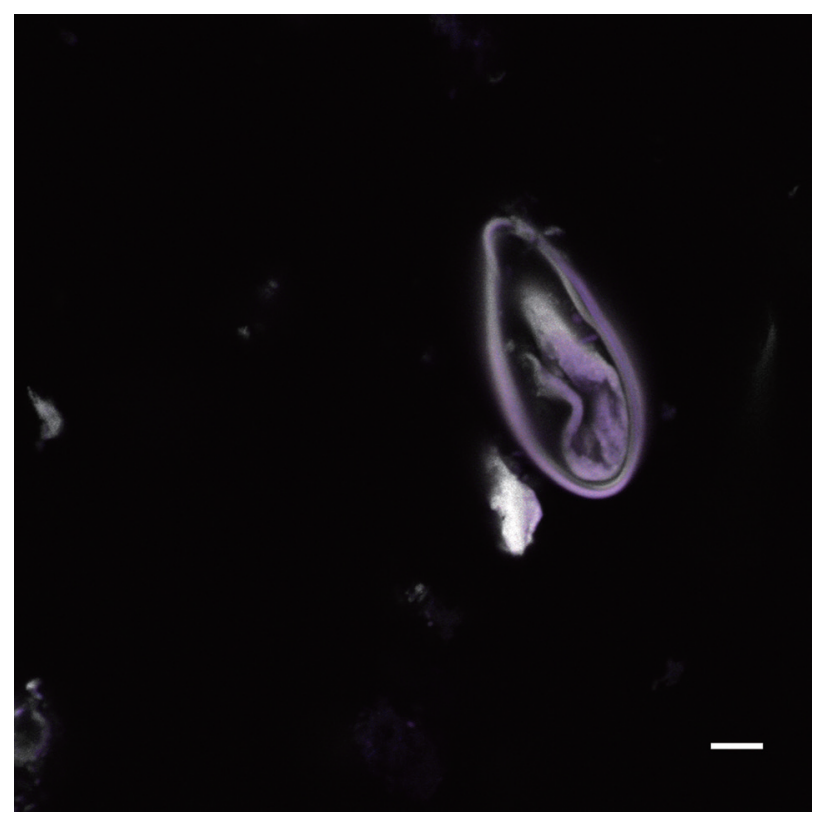

Fig. 2. Autofluorescence image of Enterobius vermicularis egg recovered from a CMC coprolite (Scale $=25 \mu \mathrm{m})$. egg specimens for several different species of parasites.

A Nikon A1 confocal laser scanning microscope (Nikon, Melville, New York, USA) with 4 photomultiplier tubes and transmitted light detector was used to detect greater morphological characters than traditional brightfield microscopy. The A1 was mounted on a Nikon 90i upright compound microscope with Nomarski Interference Contrast optics and coupled with a standard z-step motor. Single images or Z-series images were acquired with a $60 \times$ Plan Apo VC water immersion lens 1.2NA objective. Autofluorescence was excited with 4 laser lines of 405, 488, 561, and $640 \mathrm{~nm}$. Emission was detected between 425-475 $\mathrm{nm}$ pseudocolored grey, 500-550 nm pseudocolored green, 575-625 nm pseudocolored red, and 650-720 nm, respectively. Images acquisition and analysis of CLSM data was with the Nikon NIS-Elements 4.40 software supplied with the A1.

\section{RESULTS}

CLSM was used to capture images of parasite egg specimens. Among those images were photos were of Enterobius vermicularis (Figs. 1-2), a member of the trematode suborder Echinostomata (Fig. 3), Physaloptera sp. (Figs. 4-5) and Toxascaris sp. (Figs. 6-9). Channels 1 and 2 produced the most informative images by themselves; however, combined channel images

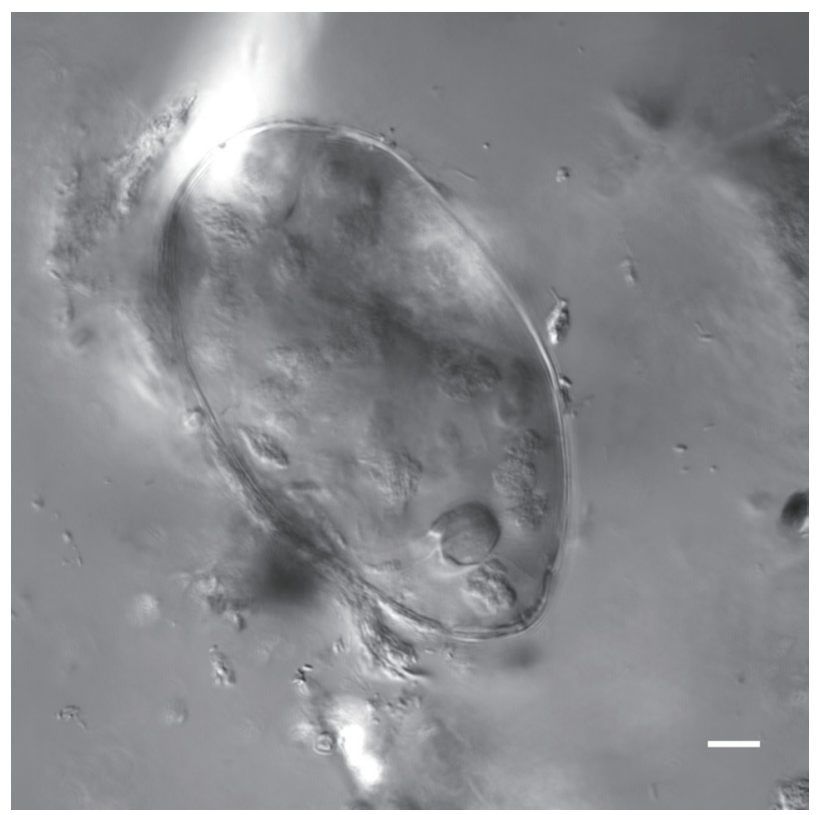

Fig. 3. Nomarski image of a parasite egg belonging to a trematode within the suborder Echinostomata recovered from a CMC coprolite (Scale $=25 \mu \mathrm{m})$. 


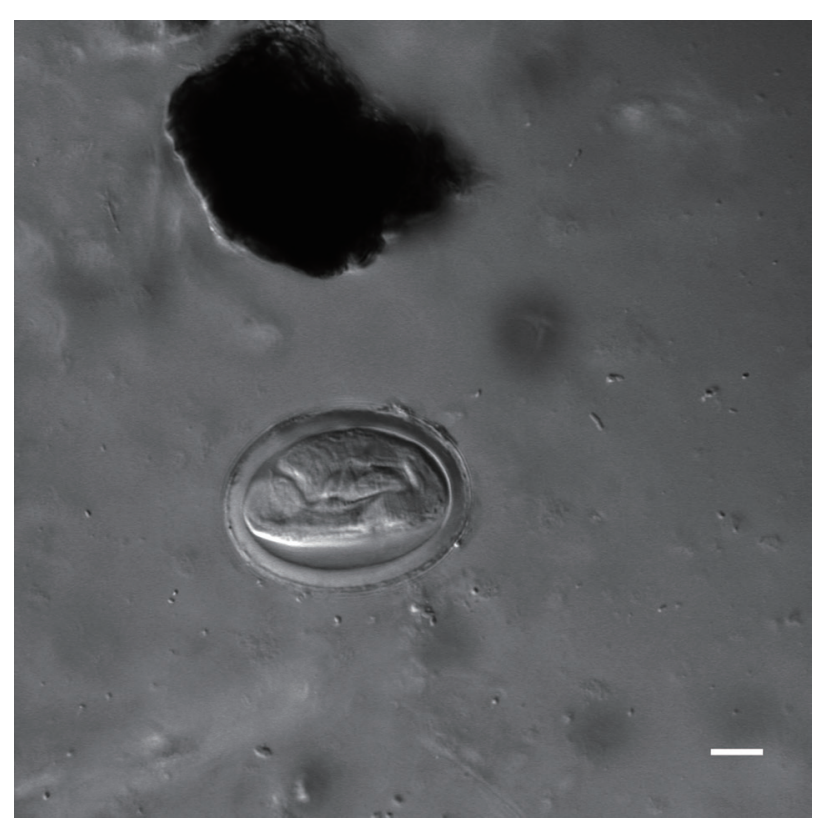

Fig. 4. Nomarski image of a Physaloptera sp. egg recovered from a CMC coprolite (Scale $=25 \mu \mathrm{m})$.

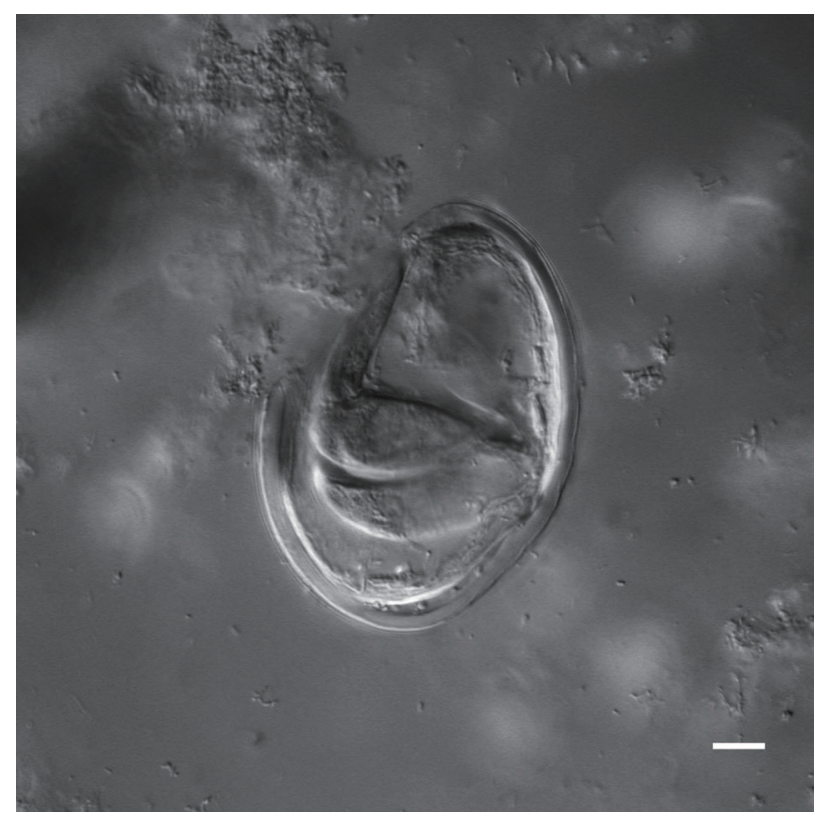

Fig. 6. Nomarski image of a Toxascaris sp. egg recovered from a CMC coprolite (Scale $=25 \mu \mathrm{m})$.

also demonstrated morphological differences that were informative. The E. vermicularis and Echinostomata specimens had been identified previously, but the CLSM images captured were informative in further supporting identification efforts.

Physaloptera sp. eggs recovered from CMC coprolites measured $45.80 \times 33.46 \mu \mathrm{m}$ on average $( \pm 2.75 \times 2.28 \mu \mathrm{m})$. They

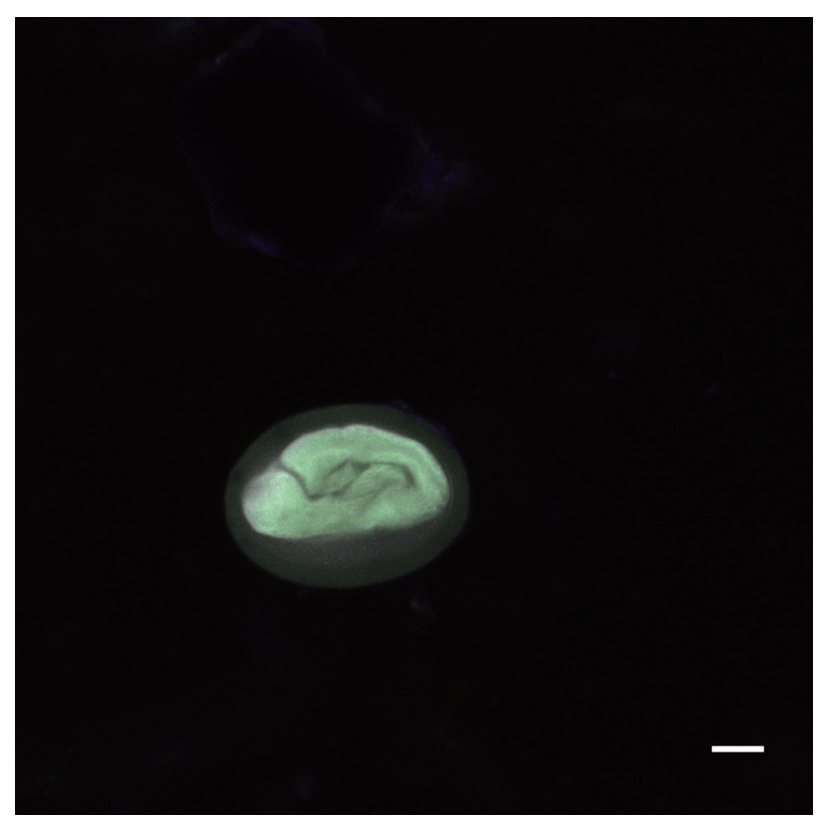

Fig. 5. Autofluorescence image of a Physaloptera sp. egg recovered from a CMC coprolite (Scale $=25 \mu \mathrm{m})$.

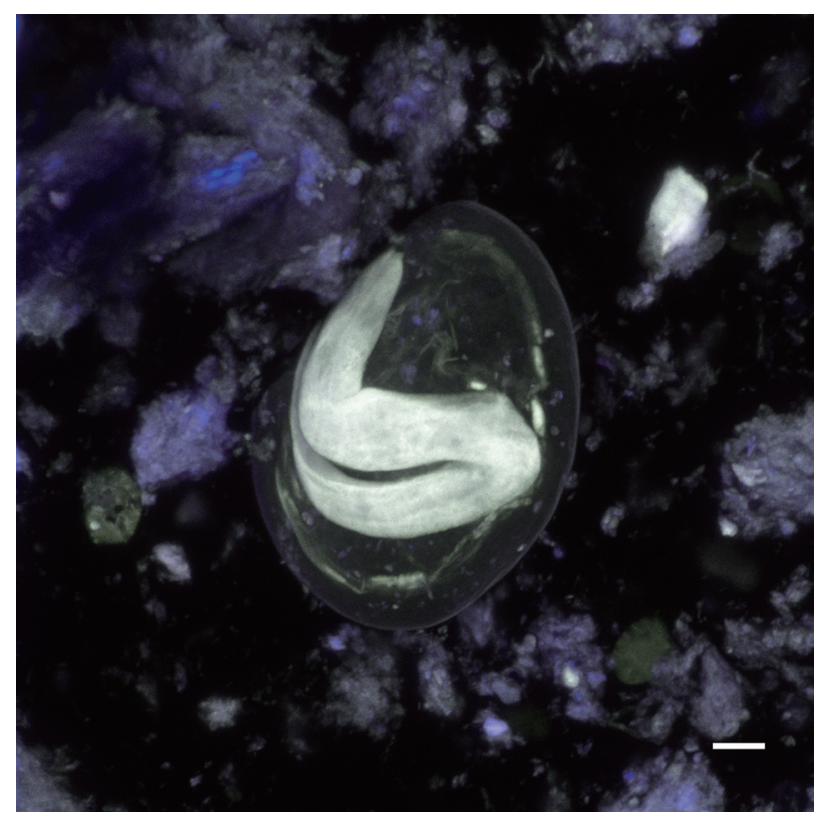

Fig. 7. Autofluorescence image of a Toxascaris sp. egg recovered from a CMC coprolite (Scale $=25 \mu \mathrm{m})$.

were oval-shaped with thick outer walls ranging between 4-6 $\mu \mathrm{m}$ in thickness, and the remains of their juvenile worms are coiled within the eggs. The Toxascaris sp. eggs recovered from CMC coprolites measured $78.80 \times 57.59 \mu \mathrm{m}$ on average $( \pm 7.60 \times 7.01 \mu \mathrm{m})$. These eggs were ellipsoid, with smooth, thick shells and most contained the remains of juveniles, 


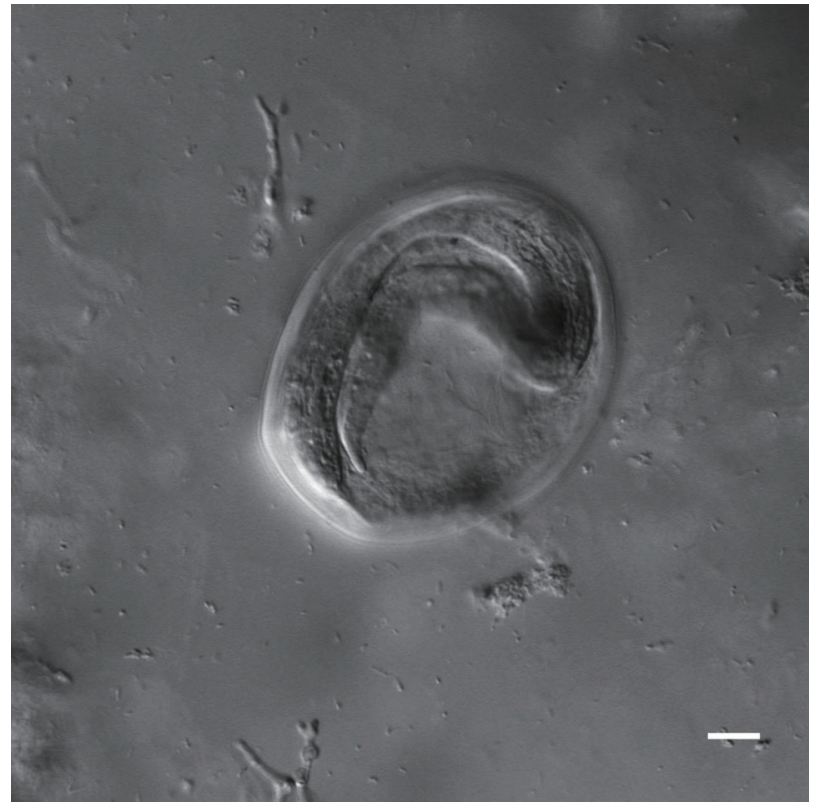

Fig. 8. Nomarski image of a Toxascaris sp. egg recovered from a $\mathrm{CMC}$ coprolite (Scale $=25 \mu \mathrm{m})$.

though some of these juveniles had emerged or were emerging at the time of death. The observable gastrointestinal tract was straight, lacking any bulbs or other diagnostic features, and the bodies of the juveniles measured approximately $220 \mu \mathrm{m}$ in length. Based on these characteristics, and upon comparisons with the morphological characters and egg size ranges of modern eggs from Ancylostoma spp., Mastophorous muris, Necator americanus, Protospirura spp., Toxascaris leonina, and Toxocara spp., it was determined that these nematode eggs were most similar to Toxascaris leonina.

\section{DISCUSSION}

This study demonstrates that CLSM is an excellent tool for archaeoparasitological studies. The CLSM images of these parasite eggs revealed detailed morphological features that were helpful in identification efforts. Many of these subtle anatomical features are less visible during LM analysis of archaeoparasitological specimens, thus using advanced microscopy techniques, such as CLSM, can be useful for determining the taxonomic designations of parasite eggs recovered from archaeological contexts [11,13]. At the time of imaging, Physaloptera sp. and Toxascaris sp. specimens had not yet been identified. The CLSM images of these specimens highlighted morphological details that were less apparent during the LM analyses and aid-

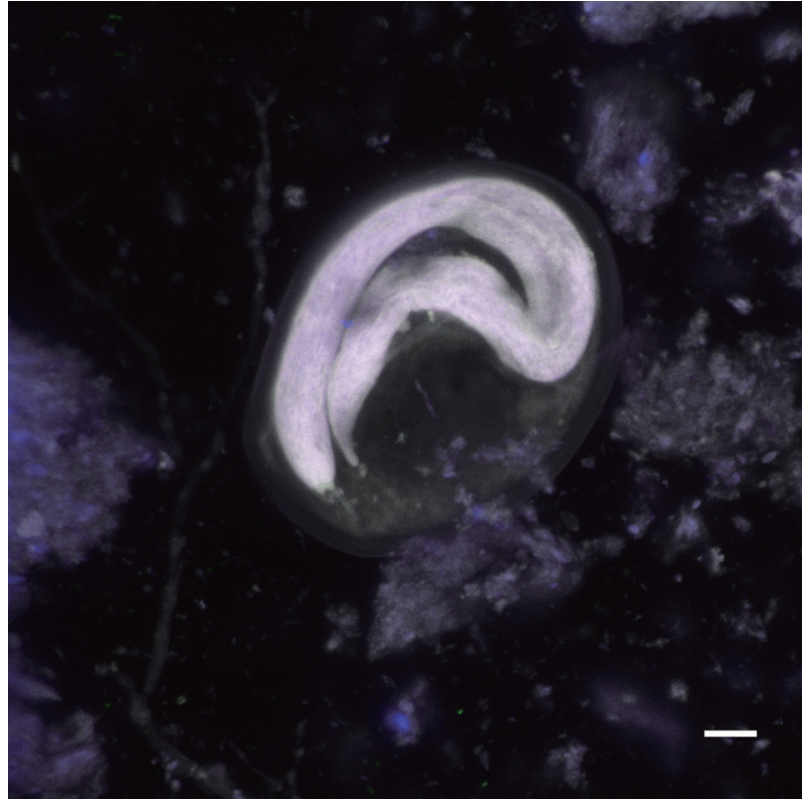

Fig. 9. Autofluorescence image of a Toxascaris sp. egg recovered from a CMC coprolite (Scale $=25 \mu \mathrm{m})$.

ed in identification efforts.

Many of the parasite specimens collected during archaeoparasitological analyses are rare and delicate, much like the archaeological materials from which they are collected. Thus, it is imperative that archaeoparasitological researchers employ care when extracting data from archaeological contexts. Some destruction of these materials is unavoidable. For example, extracting microfossil data from coprolites requires that the coprolite matrix in which these specimens are suspended be destroyed for microfossil recovery. To minimize a loss of data, researchers only use a portion of each coprolite, rather than an entire coprolite, for processing. Coprolite analysts also take many macroscopic and microscopic digital images, keep meticulous notes for later reference, and perform sequential analyses to extract as much data regarding diet and disease as possible [12]. Additionally, when samples contain large amounts of ash or other particulate contaminants that may obscure the view of parasite egg specimens, CLSM may be a useful tool for exposing the features of these specimens as such contaminants do not have autofluorescence

Given the rarity of archaeoparasitological specimens, researchers seek minimally destructive methods to preserve these specimens for subsequent analyses. To gain more detailed images of parasite specimens, scanning electron microscopy (SEM) is often employed. SEM requires specimens to be 
mounted onto adhesive stubs, and sometimes to undergo critical-point drying followed by coating the specimen in goldpalladium (sputter-coating). SEM specimen preparation techniques render specimens unusable for other types of microscopy and subsequent DNA or other molecular examinations. CLSM examinations are far less destructive to specimens. CLSM does not require permanent mounting, staining, critical-point drying, or sputter-coating prior to microscopy. While specimens that undergo CLSM are exposed to lasers, which cause some destruction on an atomic level, these specimens are still useful for subsequent analyses. This lack of additional specimen preparation prior to imaging makes CLSM well-suited for working with rare/unique specimens and specimens that could be used for further examinations, such as molecular analyses. Parasite eggs that have been imaged using CLSM can be subsequently viewed using LM, remounted in slide media if needed, or extracted for molecular analyses. This flexibility for advanced microscopy without the trade-off of specimen destruction or prevention of subsequent analysis is ideal for rare, delicate, and unique specimens, such as those used in archaeoparasitological studies.

To the authors' knowledge, this is the first instance of utilizing CLSM to aid in the identification of parasite specimens recovered from archaeological contexts. Future studies of parasite eggs, ectoparasite remains, or other unique specimens recovered from archaeological materials should consider employing CLSM in their efforts to taxonomically identify specimens and collect high-quality images without sacrificing specimens or limiting the ability to perform subsequent analyses on unique specimens.

\section{ACKNOWLEDGMENT}

The authors would like to thank Dr. Karl J. Reinhard for access to CMC coprolites and to the Pathoecology and Palynology Laboratory at the University of Nebraska-Lincoln.

\section{CONFLICT OF INTEREST}

The authors declare no conflict of interest related to this study.

\section{REFERENCES}

1. Böhm A, Bartel D, Szucsich NU, Pass G. Confocal imaging of the exo- and endoskeleton of Protura after nondestructive DNA extraction. Soil Organisms 2011; 83: 335-345.

2. Chetverikoc PE. Confocal laser scanning microscopy technique for the study of internal genitalia and external morphology of eriophyoid mites (Acari: Eriophyoidea). Zootaxa 2012; 3453: 56-68.

3. Grzywacz A, Góral T, Szpila K, Hall MJ. Confocal laser scanning microscopy as a valuable tool in Diptera larval morphology studies. Parasitol Res 2014; 113: 4297-4302.

4. Klaus AV, Schawaroch V. Novel methodology utilizing confocal laser scanning microscopy for systematic analysis in arthropods (Insecta). Integr Comp Biol 2006; 46: 207-214.

5. Lee S, Brown RL, Monroe W. Use of confocal laser scanning microscopy in systematics of insects with a comparison of fluorescence from different stains. Syst Entomol 2009; 34: 10-14.

6. Mair GR, Maule AG, Shaw C, Johnston CF, Halton DW. Gross anatomy of the muscle systems of Fasciola hepatica as visualized by phalloidin-fluorescence and confocal microscopy. Parasitology 1998; 117: 75-82.

7. Schawaroch V, Grimaldi D, Klaus AV. Focusing on morphology: Applications and implications of confocal laser scanning microscopy (Diptera: Campichoetidae, Camillidae, Drosophilidae). Proc Entomol Soc Wash 2005; 107: 323-335.

8. Valdecasas AG, Abad A. Morphological confocal microscopy in arthropods and the enhancement of autofluorescence after proteinase Kextraction. Microsc Microanal 2011; 17: 109-113.

9. Wilts EF, Wulfken D, Ahlrichs WH, Arbizu PM. The musculature of Squatinella rostrum (Milne, 1886) (Rotifera: Lepadellidae) as revealed by confocal laser scanning microscopy with additional new data on its trophi and overall morphology. Acta Zool 2012; 93: $14-27$.

10. Worsaae K, Rouse GW.The simplicity of males: dwarf males of four species of Osedax (Siboglinidae; Annelida) investigated by confocal laser scanning microscopy. J Morphol 2010; 271: 127142.

11. Daugschies A, Bialek R, Joachim A, Mundt HC. Autofluorescence microscopy for the detection of nematode eggs and protozoa, in particular Isospora suis, in swine faeces. Parasitol Res 2001; 87: 409-412.

12. Morrow JJ. Exploring parasitism in antiquity through the analysis of coprolites and quids from La Cueva de los Muertos Chiquitos, Rio Zape, Durango, Mexico. (Doctoral dissertation) [dissertation]. [Lincoln]: University of Nebraska-Lincoln. 2016.

13. Perry ES, Miller WR, Lindsay S. Looking at tardigrades in a new light: using epifluorescence to interpret structure. J Microsc 2015; 257: $117-122$. 
